\title{
Adaptive Quantum Metrology under General Markovian Noise
}

\author{
Rafał Demkowicz-Dobrzański, ${ }^{1}$ Jan Czajkowski, ${ }^{1,2}$ and Pavel Sekatski ${ }^{3}$ \\ ${ }^{1}$ Faculty of Physics, University of Warsaw, ul. Pasteura 5, PL-02-093 Warszawa, Poland \\ ${ }^{2}$ QuSoft, University of Amsterdam, Institute for Logic, Language and Computaion (ILLC), \\ P.O. Box 94242, 1090 GE Amsterdam, Netherlands \\ ${ }^{3}$ Institut fur Theoretische Physik, Universität Innsbruck, Technikerstr. 21a, A-6020 Innsbruck, Austria
}

(Received 9 May 2017; revised manuscript received 8 August 2017; published 16 October 2017)

\begin{abstract}
We consider a general model of unitary parameter estimation in the presence of Markovian noise, where the parameter to be estimated is associated with the Hamiltonian part of the dynamics. In the absence of noise, the unitary parameter can be estimated with precision scaling as $1 / T$, where $T$ is the total probing time. We provide a simple algebraic condition involving solely the operators appearing in the quantum master equation, implying, at most, $1 / \sqrt{T}$ scaling of precision under the most general adaptive quantum estimation strategies. We also discuss the requirements a quantum error-correction-like protocol must satisfy in order to regain the $1 / T$ precision scaling in case the above-mentioned algebraic condition is not satisfied. Furthermore, we apply the methods developed to understand fundamental precision limits in atomic interferometry with many-body effects taken into account, shedding new light on the performance of nonlinear metrological models.
\end{abstract}

DOI: 10.1103/PhysRevX.7.041009

Subject Areas: Atomic and Molecular Physics,

Optics, Quantum Information

\section{INTRODUCTION}

With rapid advancements in quantum optical experimental techniques, the field of quantum metrology [1-5] is entering the stage where ubiquitous quantum features of light and matter are being harnessed to deliver ultrasensitive measuring devices for real-life applications [6-12]. Along with experimental advances, theoretical foundations for the field are constantly being developed. From the first proposals of utilizing squeezed states in optical interferometry [13], through identification of fundamental limits in decoherence-free metrology [14-16], general methods have been developed, allowing us to take into account the impact of realistic decoherence effects on the performance of metrological protocols [17-24], including the most general quantum adaptive strategies $[25,26]$.

Most of the available general methods are based on the integrated form of the dynamics of a quantum system represented mathematically as a quantum channel $[20,22,25]$. This poses a serious difficulty when the dynamics is provided in terms of a master differential equation. In this case, obtaining the analytical form of the integrated dynamics is often impossible. This fact significantly limits the utility of the available methods, making it often necessary to resort to numerical calculations instead

Published by the American Physical Society under the terms of the Creative Commons Attribution 4.0 International license. Further distribution of this work must maintain attribution to the author(s) and the published article's title, journal citation, and DOI. of a more insightful analytical analysis. This deficiency has been successfully addressed in the case of single-qubit dynamics, where the full description of performance of the most general quantum metrological protocols has been given [26]. In particular, it has been shown that, provided the noise is represented by a single Pauli operator that is not proportional to the Hamiltonian itself, one can apply an error-correction procedure, allowing us to reach the Heisenberg-like, $T^{2}$, scaling of quantum Fisher information (QFI), where $T$ is the total evolution time of the probe system. On the other hand, for all other kinds of Markovian noise processes, the optimal QFI is limited by a classicallike scaling bound proportional to $T$ and hence results in a standard $1 / \sqrt{T}$ scaling of precision.

This paper provides a general solution to the problem of determining optimal performance of adaptive metrological schemes in a unitary parameter estimation problem for arbitrary Markovian dynamics. We present an explicit recipe that allows us to obtain the formulas for the behavior of QFI $F_{Q}$ in the optimal metrological protocol, based solely on the operators appearing explicitly in the master equation in the standard Gorini-Kossakowski-Lindblad-Sudarshan [27] form, with no need to integrate the dynamics whatsoever. The probe dynamics we consider is given by

$$
\frac{d \rho}{d t}=-\mathrm{i} \omega[H, \rho]+\sum_{j=1}^{J} L_{j} \rho L_{j}^{\dagger}-\frac{1}{2} \rho L_{j}^{\dagger} L_{j}-\frac{1}{2} L_{j}^{\dagger} L_{j} \rho,
$$

where $\omega$ is the frequencylike parameter to be estimated; it is associated with the unitary dynamics generated by the 
Hamiltonian $H$, while $L_{j}$ are noise operators. In particular, we show that if

$$
H \in \mathcal{S}=\operatorname{span}_{\mathbb{R}}\left\{\mathbb{1}, L_{j}^{\mathrm{H}}, \mathrm{i} L_{j}^{\mathrm{AH}},\left(L_{j}^{\dagger} L_{j^{\prime}}\right)^{\mathrm{H}}, \mathrm{i}\left(L_{j^{\prime}}^{\dagger} L_{j}\right)^{\mathrm{AH}}\right\},
$$

where $\mathrm{H}, \mathrm{AH}$ denote the Hermitian and the anti-Hermitian part of an operator, then the QFI scales, at most, linearly with $T$, and the coefficient for the bound can be obtained from a solution of a simple semidefinite program. When restricted to a single-qubit problem, this condition is equivalent to the one given in Ref. [26], requiring the noise not to be a single-rank Pauli linearly independent from the Hamiltonian. If the above linear dependence condition is not satisfied, we discuss the possibility of implementing a "quantum errorcorrection-like" protocol that yields quadratic scaling of QFI in $T$. In the qubit case, this is always possible [26] using a scheme based on preparing a maximally entangled state of the probe system and an equally dimensional ancilla. Here, we demonstrate that in higher dimensions, this is, in general, no longer the case, and this approach is not always sufficient to overcome the effects of noise.

Later, we apply the newly developed quantitative methods to determine fundamental precision bounds in atomic metrological protocols involving many-body interactions. These methods allow us, in particular, to derive, for the first time, fundamental precision bounds on nonlinear metrological protocols in the presence of decoherence. In the absence of decoherence, it is known that, in the case of the $k$-body Hamiltonian, nonlinearity may help to improve the precision scaling of QFI to $T^{2} N^{2 k}$, where $N$ is the number of atoms involved [28-36]. We show that, in the case of the $k$-body Hamiltonian and $l$-body noise, the linear dependence condition implies QFI to scale no better than $T N^{2 k-l}$ —a scaling formula identified in Ref. [37] but only for GHZ states and a limited class of noise models. Apart from determining the scaling character of the bounds, we also provide explicit coefficients for the bounds in the case of linear and nonlinear atomic interferometry in the presence of single- and two-body losses. Note that we focus here on unitary parameter estimation in the presence of noise and do not analyze the problem of estimating the noise parameter itself. This last problem, while interesting, does not enjoy equally spectacular quantum gains thanks to the use of entangled states as the unitary parameter estimation case. Often, a completely uncorrelated state proves to be optimal (for example, in the problem of estimating losses or dephasing strength), while in other cases, entanglement between a single probe and a passive ancilla is sufficient to reach optimality [38-42].

\section{FORMULATION OF THE PROBLEM}

Considering the master equation given in Eq. (1), let us denote by $\mathcal{E}_{t}^{\omega}$ the integrated form of the dynamics so that

$$
\rho_{t}^{\omega}=\mathcal{E}_{t}^{\omega}\left(\rho_{0}\right)=\sum_{j} K_{t, j}^{\omega} \rho_{0} K_{t, j}^{\omega \dagger}(t)
$$

where $K_{t, j}^{\omega}$ are Kraus operators of the evolution.

The aim is to perform optimal estimation of the $\omega$ parameter under the constraint of a fixed total evolution time $T$ under the most general adaptive quantum metrological scheme, as depicted in Fig. 1. Given the final state of the protocol $\varrho_{T}^{\omega}$, the fundamental limitation on the precision of estimating $\omega$ is given in terms of the quantum Cramér-Rao bound

$$
\Delta \omega \geq \frac{1}{\sqrt{F_{Q}}}, \quad F_{Q}=2 \sum_{a b} \frac{\left|\left\langle a\left|\dot{\varrho}_{T}^{\omega}\right| \mid b\right\rangle\right|^{2}}{\lambda_{a}+\lambda_{b}},
$$

where $F_{Q}$ is QFI for unitary encoding, the dot signifies $(d / d \omega)$, and $|a\rangle, \lambda_{a}$ are eigenvectors and eigenvalues of $\varrho_{T}^{\omega}$. In what follows, we use QFI as the figure of merit. Direct maximization of QFI of the final state over all tunable elements in the protocol, i.e., input state and controls, is a virtually impossible task unless a decoherence-free case is considered where adaptiveness is useless [16].

Luckily, provided the integrated form of the dynamics in the form of Eq. (3) is given, one can apply the methods from Refs. $[25,26]$, which allow us to obtain a universally valid upper bound on QFI valid for arbitrary adaptive strategy and hence a lower bound on uncertainty. The bound utilizes the observation that, given a quantum evolution in the form of Kraus representation (3), one can consider equivalent Kraus representation $\tilde{K}^{t, j}=$ $\sum_{j^{\prime}} u_{j j^{\prime}}^{\omega} K_{t, j^{\prime}}^{\omega}$ consisting of operators related by a unitary matrix with the original ones, or written in a more concise notation $\tilde{\mathbf{K}}=u \mathbf{K}$, where $\tilde{\mathbf{K}}=\left[\tilde{K}_{t, 0}^{\omega}, \tilde{K}_{t, 1}^{\omega}, \ldots\right]^{T}$.

The bound on QFI for the $m=T / t$ step adaptive protocol is then given in terms of minimization over different Kraus representations and reads

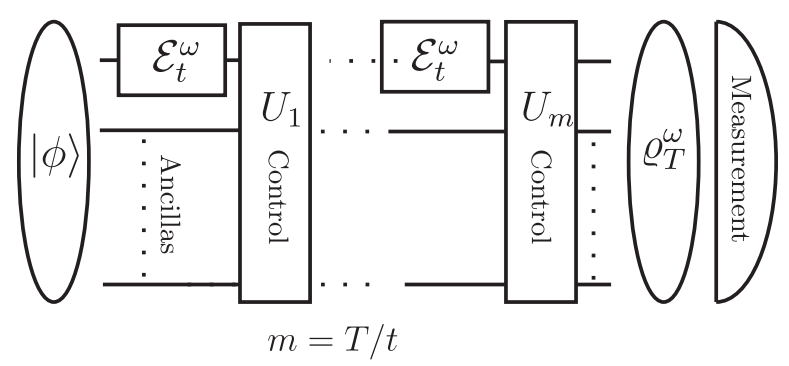

FIG. 1. General adaptive quantum metrological scheme. Total evolution time $T$ is divided into a number $m$ of $t$-long steps interleaved with general unitary controls. The collective measurement performed in the end allows us to regard this scheme as a general adaptive protocol where measurement results at some stage of the protocol influence the control actions applied at later steps. This scheme may also mimic a parallel scheme where $m$ systems in an arbitrary initial entangled state are subject to evolution through $m$ parallel channels $\mathcal{E}_{t}^{\omega}$ for a time $t$. 
$F_{Q} \leq 4 \min _{\tilde{\mathbf{K}}_{\omega}, x}\{m\|\alpha\|+m(m-1)\|\beta\|(x\|\alpha\|+\|\beta\|+1 / x)\}$,

where

$$
\alpha:=\dot{\tilde{\mathbf{K}}} \dagger \dot{\tilde{\mathbf{K}}}, \quad \beta:=-\dot{\mathbf{i}} \dot{\tilde{\mathbf{K}}}^{\dagger} \tilde{\mathbf{K}} .
$$

Here, $\|$.$\| is the operator norm, and x$ is a positive real parameter, minimization over which helps to further tighten the bound. The crucial element here is that the unitary matrix $u$ can explicitly depend on the estimated parameter $\omega$. Using the fact that $u^{\dagger} \dot{u}=-\mathrm{i} h$ for some Hermitian matrix $h$, one notes that this dependence enters the computation of $\alpha$ and $\beta$ via the substitution

$$
\dot{\tilde{\mathbf{K}}}=\dot{\mathbf{K}}-\mathrm{i} h \mathbf{K}, \quad \tilde{\mathbf{K}}=\mathbf{K} .
$$

In the most general adaptive approach, it is always advantageous to take the limit $t \rightarrow 0$, as in this case the use of adaptive gates potentially provides the greatest benefits. Note that the limit is taken only in the duration of the sensing map $\mathcal{E}_{t}^{\omega}$, and it does not influence the duration of the intermediate unitary control gates. In fact, in our model, we assume that the time needed to perform control gates is not included in the total resource count; hence, the continuous limit $t \rightarrow 0$ does not affect the control gate time. At the same time, this limit allows us to have arbitrary many control gates over the course of the sensing process and hence results in the most general adaptive strategy.

Keeping this in mind, from now on we replace $t$ with $d t$. Taking this limit, we may now utilize known relations between noise operators $L_{j}$ and the corresponding Kraus operators $K_{j}$ in order to write an explicit Kraus representation for the dynamics in the lowest order in $d t$ :

$$
\begin{gathered}
K_{0}=\mathbb{1}-\left(\frac{1}{2} \mathbf{L}^{\dagger} \mathbf{L}+\mathrm{i} H \omega\right) d t+O\left(d t^{2}\right), \\
K_{j}=L_{j} \sqrt{d t}+O\left(d t^{\frac{3}{2}}\right)(j=1, \ldots, J),
\end{gathered}
$$

where $\mathbf{L}=\left[L_{1}, L_{2}, \ldots\right]^{T}$. Note that Kraus operators' index starts at 0 , while the noise operators' index starts at 1 . This approximation correctly recovers the dynamics in the linear order in $d t$ and captures all the features of Markovian evolution.

Because of the different $d t$ scaling appearing in $K_{0}$ and $K_{j \geq 1}$ operators, it will be convenient to introduce the following structure of the matrix $h$ :

$$
h=\left[\begin{array}{c|c}
h_{00} & \mathbf{h}^{\dagger} \\
\hline \mathbf{h} & \mathfrak{h}
\end{array}\right] .
$$

Minimization over different Kraus representations in Eq. (5) now amounts to minimization over the matrix $h$, and taking into account the limit $d t \rightarrow 0$, we get

$$
F_{Q} \leq 4 \min _{h, x}\left\{T\|\alpha\| d t^{-1}+T^{2}\|\beta\| d t^{-2}(x\|\alpha\|+\|\beta\|+1 / x)\right\} .
$$

The most interesting challenge posed be the above formula is to determine the situation where $F_{Q}$ is limited by a bound scaling linearly in $T$ or where the bound scales as $T^{2}$, in which case, achieving the Heisenberg scaling may be possible.

\section{III. $H \in \mathcal{S}: T$ SCALING OF QFI}

The bound will scale linearly in $T$ if we are able to find $h$ that makes $\beta=0+O\left(d t^{2}\right)$ as well as $\alpha=$ $\alpha^{(1)} d t+O\left(d t^{2}\right)$; then by choosing $x=1 / \sqrt{d t}$, we get

$$
F_{Q} \leq 4\left\|\alpha^{(1)}\right\| T
$$

in the limit $d t \rightarrow 0$. Let us write $\beta$ explicitly in terms of $\mathbf{L}$, $h, H$ at leading orders in $d t$ :

$$
\begin{aligned}
\beta= & H d t+h_{00}\left(\mathbb{1}-\mathbf{L}^{\dagger} \mathbf{L} d t\right) \\
& +\left(\mathbf{h}^{\dagger} \mathbf{L}+\mathbf{L}^{\dagger} \mathbf{h}\right) \sqrt{d t}+\mathbf{L}^{\dagger} \mathfrak{h} \mathbf{L} d t+O\left(d t^{\frac{3}{2}}\right) .
\end{aligned}
$$

Let us denote time expansion coefficients of $h$ as follows: $h=\sum_{k=0, \frac{1}{2}, 1, \ldots} h^{(k)} d t^{k}$. We now investigate the condition $\beta=0$ order by order in time. Making $\beta=0$ in orders $O\left(d t^{0}\right)$ and $O\left(d t^{\frac{1}{2}}\right)$ implies that $h_{00}^{(0)}=h_{00}^{\left(\frac{1}{2}\right)}=0$ as well as $\mathbf{h}^{(0)}=0$. The first nontrivial condition appears in $O(d t)$ order, as this is the order where the Hamiltonian $H$ contributes and setting $h=0$ is not enough to get $\beta^{(1)}=0$. With the above substitutions, we may write the linear-order coefficient of $\beta$ :

$$
\beta^{(1)}=H+h_{00}^{(1)} \mathbb{1}+\mathbf{h}^{\dagger\left(\frac{1}{2}\right)} \mathbf{L}+\mathbf{L}^{\dagger} \mathbf{h}^{\left(\frac{1}{2}\right)}+\mathbf{L}^{\dagger} \mathfrak{h}^{(0)} \mathbf{L} .
$$

Taking into account that $h$ is Hermitian, this coefficient can be zero if and only if the Hamiltonian $H \in \mathcal{S}$, where subspace $\mathcal{S}$ is defined in Eq. (2). Analyzing the next order, we get

$$
\beta^{\left(\frac{3}{2}\right)}=h_{00}^{\left(\frac{3}{2}\right)} \mathbb{1}+\mathbf{h}^{(1) \dagger} \mathbf{L}+\mathbf{L}^{\dagger} \mathbf{h}^{(1)}+\mathbf{L}^{\dagger} \mathfrak{h}^{(1 / 2)} \mathbf{L} .
$$

We see that none of the coefficients appearing here appeared before when considering $\beta^{(1)}$, so we may put them all equal to zero, guaranteeing that $\beta=0+O\left(t^{2}\right)$ and proving the linear scaling of QFI.

In order to obtain a quantitative bound, as given in Eq. (12), let us now focus on the operator $\alpha$. Taking into 
account that $h_{00}^{(0)}=h_{00}^{\left(\frac{1}{2}\right)}=0$ as well as $\mathbf{h}^{(0)}=0$, the first nontrivial order is linear in $d t$, and the corresponding coefficient reads

$$
\alpha^{(1)}=\left(\mathbf{h}^{\left(\frac{1}{2}\right)} \mathbb{1}+\mathfrak{h}^{(0)} \mathbf{L}\right)^{\dagger}\left(\mathbf{h}^{\left(\frac{1}{2}\right)} \mathbb{1}+\mathfrak{h}^{(0)} \mathbf{L}\right) .
$$

We now need to minimize the operator norm of the above coefficient over $h$ in order to get the tightest bound:

$F_{Q} \leq 4 T \min _{\left\{h_{00}^{(1)}, \mathbf{h}^{\left(\frac{1}{2}\right)}, \mathfrak{G}^{(0)}\right\}}\left\|\alpha^{(1)}\right\|, \quad$ subject to $\beta^{(1)}=0$.

Only in some particular cases can this be done analytically. Fortunately, the above problem can be implemented as a semidefinite program, as described explicitly in Appendix A. The implementation is similar to the one presented in Ref. [22] for the Kraus operator formulation.

Since the bound (12) involves the operator norm, it may not be immediately applicable in infinite-dimensional cases where the operators appearing in the master equation are unbounded. For example, this is the case when dealing with continuous variable systems. Following the original derivation of the bound (5), however, one can refine it by taking into account some properties of the state utilized in the protocol. In particular, the states we deal with might be restricted to consist of a finite number of photons or atoms, or at least have the mean number of particles fixed. It might also be the case that, by various superselection rules, the whole Hilbert space is not available, and the bound can be tightened by analyzing the operator norm of $\alpha^{(1)}$ separately in different superselection sectors. This will amount to the calculation of operator norms on subspaces. Moreover, provided sufficient information on the time evolution of the probe state is given, the bound may even be formulated as an integral over interrogation time $T$ of a time-dependent quantity. Namely,

$F_{Q} \leq 4 \int_{0}^{T} \min \left\langle\alpha^{(1)}\right\rangle_{t} d t, \quad$ subject to $\beta^{(1)}=0$,

with $\left\langle\alpha^{(1)}\right\rangle_{t}=\operatorname{Tr} \rho_{t} \alpha^{(1)}$ setting a limit on the gain in QFI at a given instant of time, and $\rho_{t}$ the state of the system at time $t$ - see Appendix B for details on how to tighten the bound in these cases.

In what follows, it will be convenient to assume that the master equation (1) is given in the so-called canonical form $[43,44]$, where all noise operators are traceless and orthogonal, $\operatorname{Tr} L_{j}=\operatorname{Tr} L_{k}^{\dagger} L_{j}=0$ (see also Appendix C 1).

Let us now briefly comment on the single-qubit case discussed in Ref. [26]. Since $L_{i}$ operators in the canonical form are traceless, they can be written as complex combinations of Pauli matrices. To obtain a better-than-linear scaling of QFI, the noise must be a single-rank Pauli matrix linearly independent from the Hamiltonian. Mathematically, this means that there is only one $L_{j} \propto \sigma_{\vec{n}} \propto H$. Note that if we had two linearly independent $L_{j}$, they would lead to $\mathcal{S}$ being the full space of $2 \times 2$ Hermitian matrices, thanks to the fact that products $L_{j}^{\dagger} L_{j^{\prime}}$ appear in the definition of $\mathcal{S}$. Moreover, even with a single $L_{i}$ that is not proportional to a Hermitian matrix, we would have two linearly independent Hermitian matrices from its Hermitian and anti-Hermitian parts; hence the generated $\mathcal{S}$ would again be equal to the whole space of Hermitian matrices. Consequently, in the qubit case, the $H \in \mathcal{S}$ condition is equivalent to the one discussed in Ref. [26].

\section{IV. $H \notin \mathcal{S}$ : POSSIBILITY OF $T^{2}$ SCALING OF QFI}

If $H \notin \mathcal{S}$ and hence $\beta^{(1)}$ cannot be made zero, then the second term in the bound (11) will not vanish (because of $\|\beta\|^{2}$ scaling as $d t^{2}$ and canceling with the $1 / d t^{2}$ term) and will result in an upper bound scaling as $T^{2}$. This gives us hope for the Heisenberg scaling of precision. Below, we discuss the possibility to construct an adaptive quantum error-correction-inspired strategy that allows us to achieve a $T^{2}$ scaling of QFI, and we discuss some concrete examples.

In addition to the probe system, we allow for an additional ancillary system on which the evolution acts trivially. Let $\varrho=|\phi\rangle\langle\phi|$ denote the input probe-ancilla state. The adaptive protocol we consider consists of intertwining of infinitesimal-time probe evolution

$$
\mathcal{E}_{d t}^{\omega}(\varrho)=\varrho+(-\mathrm{i} \omega[H, \varrho]+\mathcal{L}(\varrho)) d t+O\left(d t^{2}\right),
$$

where $\mathcal{L}$ represents the noise part of the master equation (1) and the error-correction map $C$ applied after each infinitesimal time step $d t$. Hence, the final state of the probe and ancilla systems after the total evolution time $T$, i.e., after $T / d t$ applications of the evolution-correction step, reads

$$
\varrho_{T}^{\omega}=\mathcal{C}_{T}^{\omega}(\varrho)=\left(C \circ \mathcal{E}_{d t}^{\omega}\right)^{\circ \frac{T}{d t}}(\varrho) .
$$

Formally, in the above formula, we should write $\mathcal{E}_{d t}^{\omega} \otimes \mathcal{I}$ instead of $\mathcal{E}_{d t}^{\omega}$, as the map also acts on the ancillary system in a trivial way. From now on, for simplicity of notation, we assume that whenever an operator defined on the probe system acts on the extended probe-ancilla system, it should be understood as extended in a trivial way.

To simplify the exposition, we assume that estimation of $\omega$ is made around the $\omega_{0}=0$ point. Otherwise, by means of active control, one can always compensate for the nonzero rotation term $-\mathrm{i} \omega_{0}[H, \rho]$ in the master equation-note that, in this case, the error-correction operation $C$ may, in general, depend on $\omega_{0}$. Since QFI depends on the state and its first derivative at $\omega_{0}$ [see Eq. (4)], it is enough to consider the first-order expansion of the final state in the estimated parameter $\omega: \varrho_{T}^{\omega}=\varrho_{T}^{0}+\omega \dot{\varrho}_{T}^{0}+O\left(\omega^{2}\right)$. The zeroth-order $\varrho_{T}^{0}=\mathcal{C}_{T}^{0}(\varrho)$ is simply the action on the input state of the dynamics where the Hamiltonian part is dropped, while the first-order term 


$$
\dot{\varrho}_{T}^{0}=-\mathrm{i} \int_{0}^{T} \mathcal{C}_{T-t}^{0}\left(\left[H, \mathcal{C}_{t}^{0}(\varrho)\right]\right) d t
$$

involves terms of $\mathcal{C}_{T}^{0}$ where the Hamiltonian part of the dynamics enters linearly at different times.

Our goal is to design a protocol that protects the system from decoherence in such a way that its QFI grows quadratically in $T$ and hence mimics the performance of noiseless frequency estimation. First of all, we demand that our protocol preserves the initial state in the absence of the unitary evolution $\varrho_{t}^{0}=\mathcal{C}_{t}^{0}(\varrho)=|\phi\rangle\langle\phi|$. Furthermore, let us define a decoherence-free qubit subspace $\mathcal{H}_{Q}=\{|\phi\rangle,|\xi\rangle\}$, spanned by the input state as well as an orthogonal state $|\xi\rangle$ such that $C([H,|\phi\rangle\langle\phi|])=c(|\xi\rangle\langle\phi|-| \phi\rangle\langle\xi|), c \in \mathbb{R}$. The state on which $H$ acted is being projected back onto the subspace $\mathcal{H}_{Q}$, under the action of the error-correction step $C$. We also assume that $c \neq 0$ since otherwise the parameter $\omega$ would not be imprinted on the state at all. This allows us to simplify the expression in Eq. (21),

$$
\dot{\varrho}_{T}^{0}=\mathrm{i} c \int_{0}^{T} \mathcal{C}_{T-t}^{0}(|\phi\rangle\langle\xi|-| \xi\rangle\langle\phi|) d t .
$$

In addition, we require that the control-assisted evolution $\mathcal{C}_{T-t}^{0}$ preserves the coherence $|\phi\rangle\langle\xi|$, which leads to $\dot{\varrho}_{T}^{0}=\mathrm{i} c T(|\phi\rangle\langle\xi|-| \xi\rangle\langle\phi|)$. Calculating QFI with eigenvectors $\{|\phi\rangle,|\xi\rangle\}$ and $\lambda_{\phi}=1$ yields quadratic $F_{Q}=4 c^{2} T^{2}$ as in the case of noiseless frequency estimation. Otherwise, had the evolution damped the coherence term, resulting in $\left\|\mathcal{C}_{t}^{0}(|\phi\rangle\langle\xi|-| \xi\rangle\langle\phi|)\right\| \leq e^{-\lambda t}$, this would not be possible. Hence, in summary, the requirements for the error-correction map $C$ are the following:

(i) $C(|\phi\rangle\langle\phi|+d t \mathcal{L}(|\phi\rangle\langle\phi|))=|\phi\rangle\langle\phi|+O\left(d t^{2}\right)$,

(ii) $C(|\xi\rangle\langle\phi|+d t \mathcal{L}(|\xi\rangle\langle\phi|))=|\xi\rangle\langle\phi|+O\left(d t^{2}\right)$.

In fact, the linearity and the trace preserving properties of $C$, together with the above conditions, imply $C(|\xi\rangle\langle\xi|+d t \mathcal{L}(|\xi\rangle\langle\xi|))=|\xi\rangle\langle\xi|+O\left(d t^{2}\right)$. As a result, the requirement for QFI to grow quadratically amounts to the requirement of existence of a two-dimensional subspace protected from decoherence up to the linear order in time. We may therefore utilize known results from approximate quantum error-correction literature, which in this case reduce to the standard error-correction relation [45] for the set of error operators consisting of $L_{i}$ and the identity operator [46]:

$$
\text { (a) }\langle\phi|H| \xi\rangle \neq 0
$$

(b) $\left\langle\phi\left|L_{k}^{\dagger} L_{j}\right| \xi\right\rangle=\left\langle\phi\left|L_{j}\right| \xi\right\rangle=0$,

(c) $\left\langle\phi\left|L_{k}^{\dagger} L_{j}\right| \phi\right\rangle=\left\langle\xi\left|L_{k}^{\dagger} L_{j}\right| \xi\right\rangle$, for all $k$ and $j$, where (a) is an additional requirement that needs to be satisfied in order to keep nontrivial unitary evolution in the qubit subspace $\mathcal{H}_{Q}$. Step-by-step derivation of the above conditions is provided in Appendix C 2.

Because of the way the single-qubit error-correction protocols were applied in quantum metrology [26,47-49], a natural choice for $|\phi\rangle$ is the maximally entangled state of probe + ancilla $|\phi\rangle=(1 / \sqrt{d}) \sum_{i}|i\rangle \otimes|i\rangle$. Recall that in the canonical form of the master equation, all $\mathcal{L}$ are traceless and orthogonal, $\operatorname{Tr} L_{j}=\operatorname{Tr} L_{k}^{\dagger} L_{j}=0$ (see Appendix C 1). The Hilbert-Schmidt orthogonality of $L_{j}$ is automatically transferred to the orthogonality of $L_{j}|\phi\rangle$ vectors (where $L_{j}$ should in fact be understood here as $L_{j} \otimes \mathbb{1}$ ). We then decompose the Hamiltonian $H=H_{\perp}+H_{\|}$such that $H_{\|} \in \mathcal{S}$, while nonzero $H_{\perp} \in \mathcal{S}_{\perp}$ is orthogonal to all operators in $\mathcal{S}$. If we now take $|\xi\rangle=\left(H_{\perp}|\phi\rangle / \| H_{\perp}|\phi\rangle \|\right.$ ) (note it is, by construction, orthogonal to $|\phi\rangle$ as $H_{\perp}$ is, in particular, orthogonal to the identity operator), then one automatically satisfies the first two conditions. Condition (a) follows from $\left\langle\phi\left|H_{\perp} H\right| \phi\right\rangle \propto \operatorname{Tr} H_{\perp} H \neq 0$, while (b) follows from

$$
\left\langle\phi\left|L_{k}^{\dagger} L_{j} H_{\perp}\right| \phi\right\rangle \propto \operatorname{Tr} H_{\perp} L_{k}^{\dagger} L_{j}=0, \quad\left\langle\phi\left|L_{j} H_{\perp}\right| \phi\right\rangle=0,
$$

as $H_{\perp}$ is orthogonal to $\mathcal{S}$ with respect to the Hilbert-Schmidt scalar product. For the qubit case [26], this construction also guarantees condition (c) to be satisfied, as $H \notin \mathcal{S}$ implies only one $L_{i}=L$. Let $|0\rangle,|1\rangle$ be the eigenbasis of $H_{\perp}: H_{\perp}|i\rangle=\lambda(-1)^{i}|i\rangle\left(H_{\perp}\right.$ is orthogonal to $\mathbb{1}$ and hence has $\pm \lambda$ eigenvalues). As a result, $|\xi\rangle \propto H_{\perp}|\phi\rangle \propto(|0\rangle \otimes|0\rangle-$ $|1\rangle \otimes|1\rangle)$, and consequently, $\left\langle\phi\left|L^{\dagger} L\right| \phi\right\rangle=\left\langle\xi\left|L^{\dagger} L\right| \xi\right\rangle=$ $\operatorname{Tr}\left(L^{\dagger} L\right) / 2$. In this case, one can state that Eq. (2) is an "if and only if" condition for the impossibility of obtaining QFI scaling quadratically with $T$.

In higher dimensions, however, an error-correction scheme based on the use of the maximally entangled state of system + ancilla will not work, in general-see the Note added, which has a reference to the paper [50], where a universal construction of a quantum errorcorrection protocol satisfying all the required conditions has been provided whenever $H \notin \mathcal{S}$. In addition, this shows that the conditions that in our approach could be regarded as sufficient for quadratic scaling are actually also necessary.

\section{ATOMIC INTERFEROMETRY WITH ONE- AND TWO-BODY LOSSES}

We now demonstrate an application of the developed methods to provide bounds in atomic interferometry models where two-body effects can be placed in the noise part (two-body losses) or in the Hamiltonian part (the nonlinear metrology model). 
Let us consider a Bose-Einstein condensate (BEC) system of two-level atoms, where dynamics is described by the following master equation:

$$
\frac{\mathrm{d} \rho}{\mathrm{d} t}=-\mathrm{i} \omega\left[H^{(k)}, \rho\right]+\mathcal{L}^{(1)}(\rho)+\mathcal{L}^{(2)}(\rho),
$$

where

$$
\begin{aligned}
\mathcal{L}^{(1)}(\rho)= & \sum_{i=1}^{2} \gamma_{i}\left(a_{i} \rho a_{i}^{\dagger}-\frac{1}{2}\left\{a_{i}^{\dagger} a_{i}, \rho\right\}\right), \\
\mathcal{L}^{(2)}(\rho)= & \sum_{i=1}^{2} \gamma_{i i}\left(a_{i}^{2} \rho a_{i}^{\dagger 2}-\frac{1}{2}\left\{a_{i}^{\dagger 2} a_{i}^{2}, \rho\right\}\right) \\
& +\gamma_{12}\left(a_{1} a_{2} \rho a_{1}^{\dagger} a_{2}^{\dagger}-\frac{1}{2}\left\{a_{1}^{\dagger} a_{2}^{\dagger} a_{1} a_{2}, \rho\right\}\right)
\end{aligned}
$$

represent one-body and two-body loss processes with respective loss coefficients $\gamma_{i}, \gamma_{i j}$, and $a_{i}$ represents annihilation operators that remove an atom from the $i$ th mode. The corresponding noise operators read $L_{i}^{(1)}=\sqrt{\gamma_{i}} a_{i}$, $L_{i j}^{(2)}=\sqrt{\gamma_{i j}} a_{i} a_{j}$. We consider two different Hamiltonians that are associated with the sensing part of the dynamics:

$$
\begin{gathered}
H^{(k=1)}=\frac{1}{2}\left(a_{1}^{\dagger} a_{1}-a_{2}^{\dagger} a_{2}\right), \\
H^{(k=2)}=\frac{1}{4}:\left(a_{1}^{\dagger} a_{1}-a_{2}^{\dagger} a_{2}\right)^{2},
\end{gathered}
$$

which correspond to linear and nonlinear metrological scenarios. For clarity of presentation, we put the normal ordering operation in the definition of $H^{(2)}$ in order to make sure that we take into account only terms that appear from the interaction between two different particles.

Let us start with the linear Hamiltonian case $k=1$ but keep both the single- and two-body losses. This kind of model is well tailored to analyze metrological BEC experiments such as magnetometry experiments using spin-squeezed BEC [10]. Let us calculate $\beta^{(1)}$ according to Eq. (14):

$$
\begin{aligned}
\beta^{(1)}= & \frac{1}{2}\left(a_{1}^{\dagger} a_{1}-a_{2}^{\dagger} a_{2}\right)+h_{00}^{(1)} \mathbb{1}+\left(\sum_{i} \sqrt{\gamma_{i}} h_{i}^{\left(\frac{1}{2}\right)} a_{i}+\text { H.c. }\right) \\
& +\sum_{i j} \mathfrak{h}_{i j}^{(0)} \sqrt{\gamma_{i} \gamma_{j}} a_{i}^{\dagger} a_{j}+\ldots .
\end{aligned}
$$

We now look at the possibility of choosing entries of $h$ so that we can set the above quantity to zero. Note that we have not written explicitly the noise operators related to two-body losses because operators related to twobody losses appearing in the above equation would be of the form $a_{i} a_{j}, a_{i}^{\dagger} a_{j}, a_{i}^{\dagger} a_{j}^{\dagger} a_{i}^{\prime} a_{j}^{\prime}$ and would be linearly independent on the operators appearing in the Hamiltonian part. Hence, trying to set $\beta^{(1)}=0$, we need to focus on one-body-loss operators only. We are free to put all coefficients of $h$ in front of terms related to two-body losses equal to zero. If we succeed in setting $\beta^{(1)}=0$ using only one-body-loss operators, this will also imply that two-body losses are irrelevant in trying to assess the fundamental precision limit on frequency estimation in this case. By inspecting Eq. (34), it is clear that we can make $\beta^{(1)}=0$ by choosing $h_{00}^{(1)}=0, \quad h_{i}^{\left(\frac{1}{2}\right)}=0$, $\mathfrak{h}_{11}^{(0)}=-\frac{1}{2} \gamma_{1}^{-1}, \mathfrak{h}_{22}^{(0)}=\frac{1}{2} \gamma_{2}^{-1}, \mathfrak{h}_{12}^{(0)}=0$.

We should remember, however, that when deriving the final bound using Eq. (16), we face the problem that operators appearing under the operator norm are unbounded; hence, the bound will formally be infinite and thus useless. Physically, this is due to the fact that we have not set any constraints on the number of atoms we use in the experiment. From now on, we assume we have $N$ atoms at our disposal, and at every adaptive step, we replace the lost atoms with new ones, keeping the number of atoms constant. We discuss this approach in detail in Appendix B and argue that, by doing so, we do not lose generality of our bounds. Thanks to this, we are able to write $a_{1}^{\dagger} a_{1}+a_{2}^{\dagger} a_{2}=$ $N 1$ when calculating the operator norm of $\alpha^{(1)}$, remembering that, in the end, we operate in a fixed particle-number subspace.

Let us now go back to Eq. (34). With the fixed particlenumber constraint imposed, $a_{1}^{\dagger} a_{1}, a_{2}^{\dagger} a_{2}$ and $\mathbb{1}$ are no longer independent operators. This gives us an additional freedom in choosing coefficients of $h$ in order to keep $\beta^{(1)}=0$; namely, we can take $h_{00}^{(1)}=-N \xi, \mathfrak{h}_{11}^{(0)}=\gamma_{1}^{-1}\left(\xi-\frac{1}{2}\right), \mathfrak{h}_{22}^{(0)}=$ $\gamma_{2}^{-1}\left(\xi+\frac{1}{2}\right)$, with $\xi$ being a free parameter. The QFI bound can now be obtained by minimizing $\|\alpha\|$ over $\xi$ :

$F_{Q} \leq T \min _{\xi}\left\|\gamma_{1}^{-1}(2 \xi-1)^{2} a_{1}^{\dagger} a_{1}+\gamma_{2}^{-1}(2 \xi+1)^{2} a_{2}^{\dagger} a_{2}\right\|$.

The operators $a_{1}^{\dagger} a_{1}, a_{2}^{\dagger} a_{2}$ commute, and their common basis is $|n, N-n\rangle$ (where $n$ is the number of atoms in mode 1); hence, in the above minimization, we can replace $a_{1}^{\dagger} a_{1}$ with $n$ and $a_{2}^{\dagger} a_{2}$ with $N-n$ :

$F_{Q} \leq T \min _{\xi} \max _{0 \leq n \leq N} \gamma_{1}^{-1}(2 \xi-1)^{2} n+\gamma_{2}^{-1}(2 \xi+1)^{2}(N-n)$.

The minimum is achieved for $\xi$, which satisfies $\gamma_{1}^{-1}(2 \xi-1)^{2}=\gamma_{2}^{-1}(2 \xi+1)^{2}$, and it reads

$$
F_{Q} \leq \frac{4 T N}{\left(\sqrt{\gamma_{1}}+\sqrt{\gamma_{2}}\right)^{2}} .
$$


This bound indeed agrees with a known bound of $N$ particle interferometry, with losses [20,22,51,52]

$$
F_{Q} \leq \frac{4 T t N}{\left(\sqrt{\frac{1-\eta_{1}}{\eta_{1}}}+\sqrt{\frac{1-\eta_{2}}{\eta_{2}}}\right)^{2}},
$$

where $\eta_{i}=e^{-\gamma_{i} t}$ after taking the limit $t \rightarrow 0$. Note, however, that the derivation presented in this paper did not require any educated guess [20] or a numerically indicated optimal form of the Kraus representation [22], but it resulted in a purely algebraic analysis of the noise operators and the Hamiltonian appearing in the master equation. Moreover, when deriving the bound, we could clearly see that two-body losses do not have an impact on the bound.

We now study the fundamental bounds in a nonlinear metrological model with $k=2$, in which case

$$
\begin{aligned}
\beta^{(1)}= & \frac{1}{4}\left(a_{1}^{\dagger 2} a_{1}^{2}+a_{2}^{\dagger 2} a_{2}^{2}-2 a_{1}^{\dagger} a_{2}^{\dagger} a_{1} a_{2}\right)+h_{00}^{(1)} \mathbb{1} \\
& +\mathfrak{h}_{11,11}^{(0)} \gamma_{11} a_{1}^{\dagger 2} a_{1}^{2}+\mathfrak{h}_{22,22}^{(0)} \gamma_{22} a_{2}^{\dagger 2} a_{2}^{2} \\
& +\mathfrak{h}_{12,12}^{(0)} \gamma_{12} a_{1}^{\dagger} a_{2} \dagger a_{1} a_{2}+\ldots,
\end{aligned}
$$

where we explicitly wrote only terms relevant for further discussion. In particular, we can ignore the onebody-loss operators, as they are linearly independent from the Hamiltonian and hence will not contribute to the bound. Similarly, as in the linear case, we assume that we are dealing with $N$-atom states. Hence, we utilize the fact that $\left(a_{1}^{\dagger} a_{1}+a_{2}^{\dagger} a_{2}\right)^{2}=N^{2} \mathbb{1}$, implying the following relation: $a_{1}^{\dagger 2} a_{1}^{2}+a_{2}^{\dagger} a_{2}^{2}+2 a_{1}^{\dagger} a_{2}^{\dagger} a_{1} a_{2}=N(N-1) \mathbb{1}$, which allows us to again introduce a free parameter $\xi$ into coefficients of $h: \mathfrak{h}_{11,11}^{(0)}=\frac{1}{4} \gamma_{11}^{-1}(\xi-1), \mathfrak{h}_{22,22}^{(0)}=\frac{1}{4} \gamma_{22}^{-1}(\xi-1)$, $\mathfrak{h}_{12,12}^{(0)}=\frac{1}{2} \gamma_{12}^{-1}(\xi+1), h_{00}^{(1)}=-\frac{1}{4} \xi N(N-1)$. Using Eq. (5), we arrive at the following bound:

$$
\begin{aligned}
F_{Q} \leq & \min _{\xi} \frac{T}{4} \| 2 \gamma_{12}^{-1}(\xi+1)^{2} N(N-1) \mathbb{1} \\
& +a_{1}^{\dagger 2} a_{1}^{2}\left(\gamma_{11}^{-1}(\xi-1)^{2}-2 \gamma_{12}^{-1}(\xi+1)^{2}\right) \\
& +a_{2}^{\dagger 2} a_{2}^{2}\left(\gamma_{22}^{-1}(\xi-1)^{2}-2 \gamma_{12}^{-1}(\xi+1)^{2}\right) \| .
\end{aligned}
$$

Since all operators under the operator norm commute and have a common eigenbasis $|n, N-n\rangle$, we can write the above bound explicitly, replacing $a_{1}^{\dagger 2} a_{1}^{2}$ with $n(n-1)$ and $a_{2}^{\dagger 2} a_{2}^{2}$ with $(N-n)(N-n-1)$. Calculating the operator norm now amounts to maximization over $n$. In the case $\gamma_{11}=\gamma_{22}$, the above problem has an explicit solution, which in the limit of large $N$ reads

$$
F_{Q} \leq \frac{N^{2} T}{\gamma_{12}}\left\{\begin{array}{ll}
\frac{2}{(1+\sqrt{\lambda})^{2}} & \lambda \geq 1 \\
\frac{1}{1+\lambda} & \lambda<1
\end{array},\right.
$$

where $\lambda=2 \gamma_{11} / \gamma_{12}$. To the best of our knowledge, this is the first example of a fundamental bound in the nonlinear metrology model, taking into account many-body decoherence effects. Details of the above derivation, as well as the discussion of the case $\gamma_{11} \neq \gamma_{22}$, are presented in Appendix D.

\section{QUANTUM METROLOGY WITH GENERAL MANY-BODY INTERACTIONS}

Let us now investigate what can be said, in general, concerning the fundamental bounds in nonlinear metrology models with many-body interactions, without specifying the actual form of the dynamics but just its nonlinear character and trying to identify the resulting characteristic scaling of QFI.

Consider a system of $N$ atoms, where the Hamiltonian part is a result of $k$-body interactions, while the noise part is of the $l$-body type. To be more specific, we consider the dynamics of the form

$$
\begin{aligned}
\frac{\mathrm{d} \rho}{\mathrm{d} t}= & -\mathrm{i} \omega\left[\sum_{\nu \in \Upsilon_{k}} H_{\nu}, \rho\right]+\gamma \sum_{\mu \in \Upsilon_{l, j}} L_{\mu, j} \rho L_{\mu, j}^{\dagger}-\frac{1}{2} \rho L_{\mu, j}^{\dagger} L_{\mu, j} \\
& -\frac{1}{2} L_{\mu, j}^{\dagger} L_{\mu, j} \rho,
\end{aligned}
$$

where $\Upsilon_{k}=\left\{\left(i_{1}, \ldots, i_{k}\right)\right\}$ represents all $k$ element combinations of the $N$ element set, and the operator index $\nu \in \Upsilon_{k}$ denotes particles that a given operator acts on. We have also introduced a positive coefficient $\gamma$ in order to be able to discuss effects of rescaling the noise strength. In what follows, we assume that $k, l \ll N$. This is a general scenario considered in the field of nonlinear metrology [28-36] but, very often, without the noise part. Including the noise part in this form in the analysis is extremely challenging and has only been analyzed for very specific noise models and only in the case of input GHZ states [37].

Clearly, plugging all operators directly into formulas for $\alpha$ and $\beta$ would make the problem intractable in the case of large $N$. We show that it is possible to apply our tools effectively to the dynamics of $n \geq \max (k, l), n \ll N$ atoms, and from this analysis, we can infer the final scaling for the whole system of $N$ atoms. Recall that while investigating the fundamental limits to adaptive schemes, we always consider the limit $t \rightarrow 0$, and the only relevant order of the dynamics we need to take into account is the linear one. Hence, we may replace the original dynamics as represented by Eq. (42) with a scheme where each $n$-tuple of particles experiences the dynamics sequentially. By a Trotter expansion argument, this will only introduce an $O\left(t^{2}\right)$ difference because of a potential lack of commutation of the operators acting on different subsets of particles (see Fig. 2). This represents the dynamics in terms of elementary operations $\varepsilon_{t}^{\omega^{\prime}, \gamma^{\prime}}$ acting on $n$ particles only, which we refer to as subchannels. In the above scheme, the 


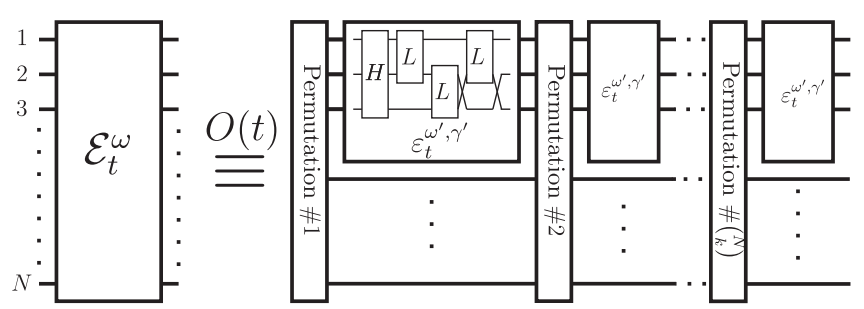

FIG. 2. Equivalent (up to linear order in $t$ ) representation of the $N$-particle dynamics in the form of a subsequent action of the $k$-particle Hamiltonian $H$ and $l$-particle noise $L$ on all $n$-particle subsets of the total $N$ particles (the circuit is given for $n=k=$ $3 \geq l=2$ ). Since the number of applications of the noise part is enhanced here by a factor $\chi_{l} \propto N^{n-l}$, we need to rescale the noise coefficient in the above scheme to $\gamma^{\prime} \propto \gamma / \chi_{l}$ to preserve the equivalence. Similarly, if $n>k$, we would need to rescale the frequency parameter to $\omega^{\prime}=\omega / \chi_{k}$. This representation allows us to calculate the bound on QFI for the whole dynamics by analyzing the properties of an elementary $n$-particle subchannel $\varepsilon_{t}^{\omega^{\prime}, \gamma^{\prime}}$.

$H$ box denotes a free unitary evolution of $k$ particles for a time $t$, while the $L$ box represents the noisy part of the dynamics, which also lasts for a time $t$. In order to keep the equivalence to the original problem, we need to rescale the noise coefficient $\gamma^{\prime}=\gamma / \chi_{l}$, where $\chi_{l}=$ $\left(\begin{array}{l}N \\ n\end{array}\right)\left(\begin{array}{l}n \\ l\end{array}\right) /\left(\begin{array}{l}N \\ l\end{array}\right) \propto N^{n-l}$. The rescaling is necessary since the number of noisy gates applied is enhanced by a factor of $\chi_{l}$ compared with the original dynamics. Similarly, we need to modify the Hamiltonian evolution by rescaling $\omega^{\prime}=\omega / \chi_{k} \propto$ $\omega N^{-(n-k)}$-in the example of Fig. 2, this is not necessary since $k=n$.

Let us assume that it is possible to find an $h_{\varepsilon}$ that makes $\beta_{\varepsilon}=0+O\left(t^{2}\right)$, where $\beta_{\varepsilon}$ should be understood as the $\beta$ operator corresponding to the elementary dynamics $\varepsilon_{t}^{\omega^{\prime}, \gamma^{\prime}}$. This again corresponds to the situation where the Hamiltonian $H$ belongs to $\mathcal{S}_{\varepsilon}$, which is constructed from noise operators entering $\varepsilon^{\omega^{\prime}, \gamma^{\prime}}$ according to Eq. (2). We can now apply the bound (11), treating $\varepsilon_{t}^{\omega^{\prime}, \gamma^{\prime}}$ as the fundamental building block for the adaptive strategy; since there are now $\left(\begin{array}{c}N \\ n\end{array}\right) T / t$ such elementary blocks, we arrive at $F_{Q} \leq 4\left(\begin{array}{l}N \\ n\end{array}\right)\left\|\alpha_{\varepsilon^{\left(\omega^{\prime}, \gamma^{\prime}\right.}}^{(1)}\right\| T$.

Let us inspect Eqs. (14) and (16) in order to understand the impact of the rescaling factors $\chi_{l}, \chi_{k}$ on the value of the above bound. Rescaling of $\gamma$ introduces an additional $1 / \sqrt{\chi_{l}}$ factor to all $L$ operators. Additionally, taking into account that the Hamiltonian is rescaled by the $1 / \chi_{k}$ factor, according to Eq. (14), in order to satisfy the $\beta^{(1)}=0$ constraint, $\mathfrak{h}^{(0)}$ needs to be rescaled by $\chi_{l} / \chi_{k}$ while $\mathbf{h}^{\left(\frac{1}{2}\right)}$ by $\sqrt{\chi_{l}} / \chi_{k}$ factors.

Together with Eq. (16), this implies that $\alpha^{(1)}$ is rescaled by $\chi_{l} / \chi_{k}^{2}$. Therefore, we finally arrive at

$$
F_{Q} \leq 4 T\left\|\alpha_{\varepsilon^{\omega,, y}}^{(1)}\right\|\left(\begin{array}{l}
N \\
n
\end{array}\right) \frac{\chi_{l}}{\chi_{k}^{2}} \propto T N^{2 k-l}
$$

Note that the obtained scaling agrees with what we have obtained in the models discussed in Sec. V. Thus, the case where $\beta^{(1)}$ could be zero corresponds to either $n=1$, $k=1, l=1$ or $n=2, k=2, l=2$, and indeed, we obtain, respectively, $T N$ and $T N^{2}$ scalings. This shows that the general approach based on splitting the complex multiparticle dynamics into small subchannels involving only the number of particles required to model a given degree of nonlinearity is sufficient to obtain a proper scaling of the precision bounds. This general approach can also be utilized to obtain quantitative bounds, which will be the subject of a separate publication [53].

The $N^{2 k-l}$ scaling of QFI, or equivalently $N^{-(k-l / 2)}$ scaling of parameter estimation precision, was also observed in Ref. [37] for protocols based on utilizing the GHZ class of states and models where all $H$ and $L_{i}$ operators commute. Our approach proves the scaling for both the most general class of states and for adaptive strategies. Once our bound can be derived (i.e., $\beta^{(1)}$ can be set to zero), we can claim that, in models considered in Ref. [37], indeed the GHZ and product states provide the optimal scaling. In the more general approach, however, where arbitrary states and adaptive strategies are allowed, this will not necessarily be the case.

To show both the power and simplicity of our approach, let us therefore consider the class of dynamics, which we refer to as nonlinear metrology with multiparticle dephasing, where $L_{i}$ and $H$ commute as considered in Ref. [37], and try to apply our methods to derive general precision bounds in this case. We take $H_{\nu}=\sigma_{z}^{\nu_{1}} \otimes \ldots \otimes \sigma_{z}^{\nu_{k}}$ and $L_{\mu}=\sigma_{z}^{\mu_{1}} \otimes \ldots \otimes \sigma_{z}^{\mu_{l}}$. Let us inspect the structure of the subspace $\mathcal{S}$ [see Eq. (2)] and invoke the representation of the dynamics in terms of subchannels $\varepsilon$ as depicted in Fig. 2. We look for what $k, l$ we can satisfy the $H \in \mathcal{S}_{\varepsilon}$ condition. The obvious case is $k=l$ when we simply consider $n=k=l$ subchannels, in which case, the operator $H$ is proportional to the operator $L$. Similarly, we can show that $H \in \mathcal{S}$ if $k=2 l$ since if we take $n=k$ we can obtain $H$ from products of $L$ acting on two separate subsets of $l$ particles. More generally, provided $k$ is even and $k \leq 2 l$, we can consider $n=l+k / 2$ particle subchannels and obtain $H$ by multiplying $L$ acting on two sets of $l$ particles where $(l-k / 2)$ of them overlap. Thus, the product of two $\sigma_{z}$ on overlapping particles is the identity, and we can obtain a $2 l-2(l-k / 2)=k$ fold tensor product of $\sigma_{z}$ acting on the remaining particles.

In particular, for nonlinear metrology $k=2$ and linear dephasing $l=1$, we automatically get the $F_{Q} \lesssim T N^{3}$ bound, while for the two-body Hamiltonian $k=2$ and nonlinear dephasing $l=2$, we get $F_{Q} \lesssim T N^{2}$, proving the fundamental character of the scaling obtained in Ref. [37]. However, if we take $k=1$ and $l=2$, then according to our approach, $H \notin \mathcal{S}_{\varepsilon}$-as we cannot obtain a single $\sigma_{z}$ from products of two or four $\sigma_{z}$ that appear in the definition of 
$\mathcal{S}_{\varepsilon}$. Note that, indeed, in this case, the subspace spanned by $|0\rangle^{\otimes N} \pm|1\rangle^{\otimes N}$ is actually immune to decoherence as all $L_{i}$ operators involve the product of two $\sigma_{z}$ acting on different sites and therefore yield a trivial 1 factor when acting on states from this subspace. Still, the single-body Hamiltonian acts nontrivially, and we get $F_{Q} \propto T^{2} N^{2}$, showing the possibility of better-than-linear scaling both in $N$ and in $T$ and demonstrating that, indeed, in this case the bound $F_{Q} \lesssim T N^{2 k-l} \propto T$ is invalid.

Let us also comment here on the issue of optimization of quantum metrological protocols under the fixed definite particle number or just the mean number of particles fixed. In linear metrology, QFI scales quadratically in the decoherence-free case, and hence, maximization of QFI under fixed mean particle number may lead to surprising conclusions for the possibility of beating Heisenberg scaling or even reaching arbitrarily high values (see, e.g., Ref. [54]). This should be understood as a deficiency of the QFI figure of merit, which, in general, provides only a lower bound on achievable uncertainty via the CramérRao bound, whereas saturability of the bound requires more detailed arguments [34,55-57]. If decoherence makes QFI scale linearly with the number of particles, though, this issue becomes nonexistent, and one can replace fixed particle numbers with mean particle numbers in the formulas for the bounds $[3,23,58]$. In the nonlinear models discussed above, even in the presence of decoherence, the bounds will, in general, scale superlinearly with particle numbers; hence, again, the task of maximizing QFI under the fixed mean particle number will be ill-posed, and as a result, a meaningful discussion of such problems would require going beyond the QFI paradigm.

\section{CONCLUSIONS AND DISCUSSION}

Throughout this work, we have assumed Markovian semigroup dynamics. Validity of this approximation requires, in particular, coarse graining of the evolution of the system on time scales larger than the environmental characteristic relaxation time scales. Therefore, the "continuous limit" $t \rightarrow 0$ that we have adopted while deriving our bounds should be understood as the limit of very short times but still in the regime where the Markovian approximation holds. Consequently, the fundamental character of the bounds we derive hinges upon the assumption that one cannot operate on time scales shorter than the ones characteristic for the Markovian approximation. If this assumption is dropped, one may expect different time scalings of QFI (see, e.g., Refs. [59-61]). Still, combining the framework of the most general adaptive protocols with non-Markovian or even Markovian nonsemigroup dynamics is a nontrivial task. In particular, focusing solely on the reduced system dynamics may not be, in general, sufficient to describe the effects of control operations acting on the system, and more information on the system + environment interaction may be required. Moreover, in such scenarios, one cannot a priori justify the framework where the time used up by control operations is not relevant, or argue that a model with infinitely many control operations is the optimal one. We feel that providing a general framework to study the potential of adaptive quantum metrological protocols in the presence of non-Markovian noise goes beyond the direct generalization of the methods of this paper, and we leave this task for future research.

Another way of generalizing the results of this work is to assume the environment can be partially monitored, which may open up completely new possibilities of fighting decoherence. It is also interesting to study multiparameter estimation scenarios where adaptiveness seems to already play some role at the decoherence-free level [62].

To conclude, we have provided a simple algebraic criterion, Eq. (2), determining, at most, linear time scaling of QFI in the estimation of a unitary parameter under general Markovian dynamics. Its high utility stems from the fact that it deals directly with the Hamiltonian and noise operators appearing in the master equation and does not require integration of the dynamics. We have shown how the bounds can be derived in atomic interferometric models involving many-body interactions. In the qubit case, we showed that the condition (2) is sufficient and necessary for fundamental linear time scaling of QFI. In the case of arbitrary dynamics in arbitrary dimensions, however, it has only been proven to be a sufficient condition. Whether it is also a necessary one remains an open question.

\section{ACKNOWLEDGMENTS}

We thank Wolfgang Dür, Janek Kołodyński, Animesh Datta, Philipp Treutlein, and Krzysztof Pawłowski for fruitful discussions. This work was supported by the Polish Ministry of Science and Higher Education Iuventus Plus program for years 2015-2017, Grant No. 0088/IP3/2015/73, National Science Center (Poland) Grant No. 2016/22/E/ST2/00559, and Swiss National Science Foundation Grant No. P300P2_167749. J. C. acknowledges support by the Netherlands Organisation for Scientific Research (NWO) VIDI grant (Project No. 639.022.519).

Note added.-Recently, Ref. [50] appeared, where, in addition to the independent derivation of the results presented in Sec. III of our paper, an explicit construction of the quantum error-correction protocol has been provided, yielding $T^{2}$ scaling of the QFI whenever condition (2) is not satisfied. As a result, this paper answered the open problem stated above on whether the condition (2) is indeed the if and only if condition for the impossibility of preserving the $T^{2}$ scaling of QFI via the most general quantum protocols in the presence of noise for systems of arbitrary dimensions. Note also that our conditions for an 
effective error-correction protocol [Eqs. (25)-(27)] are equivalent to the ones provided in Ref. [50]. In particular, by expressing the projector $\Pi_{C}$ from Ref. [50] using $|\phi\rangle,|\xi\rangle$ from Sec. IV of our paper as $\Pi_{C}=|\phi\rangle\langle\phi|+| \xi\rangle\langle\xi|$, we see that their condition (15) is equivalent to ours [Eqs. (26) and (27)], while their condition (17) is equivalent to our Eq. (25) - on the one hand, we can always find two states in this subspace $\Pi_{C}$ that are coupled by the Hamiltonian; on the other hand, if two states from the subspace are coupled, the action of the Hamiltonian is nontrivial. Finally, let us briefly describe how the universal error-correction code of Ref. [50] appears in our language. The key idea is to use the spectral decomposition of the part of the Hamiltonian perpendicular to the noise space $H_{\perp}=P-Q$, where $P$ and $Q$ are orthogonal and positive semidefinite, and $\operatorname{Tr} P=$ $\operatorname{Tr} Q$ since $H_{\perp}$ is traceless by construction. Next, on the probe-plus-ancilla system, we define the corresponding purification states $|p\rangle$ and $|q\rangle$, satisfying

$$
P=h_{\perp} \operatorname{Tr}_{A}|p\rangle\langle p| \quad \text { and } \quad Q=h_{\perp} \operatorname{Tr}_{A}|q\rangle\langle q|,
$$

where $h_{\perp}>0$ since $H_{\perp}$ is nonzero. Then, the virtual qubit states of Sec. IV can be defined as $|\phi\rangle=(1 / \sqrt{2})(|p\rangle+|q\rangle)$ and $|\xi\rangle=(1 / \sqrt{2})(|p\rangle-|q\rangle)$.

\section{APPENDIX A: CALCULATING THE BOUND VIA SEMIDEFINITE PROGRAMMING}

Given that the condition $H \in \mathcal{S}$ is satisfied, we know that the scaling of QFI is bound to be linear in $T$. In order to obtain the tightest bound of the form (12), we need to minimize the operator norm of $\alpha^{(1)}$, keeping the constraint $\beta^{(1)}=0$. First, we construct a matrix $A$,

$$
A=\left[\begin{array}{cc}
\sqrt{\lambda} \mathbb{1} & \mathbf{h}^{\left(\frac{1}{2}\right) \dagger} \mathbb{1}+\mathbf{L}^{\dagger} \mathfrak{h}^{(0)} \\
\mathbf{h}^{\left(\frac{1}{2}\right)} 1+\mathfrak{h}^{(0)} \mathbf{L} & \sqrt{\lambda} \mathbb{1}^{\otimes J}
\end{array}\right] .
$$

Minimizing the operator norm $\left\|\alpha^{(1)}\right\|$ is now equivalent to minimizing $\lambda$ subject to $A \geq 0$, with the additional constraint coming from the equation $\beta^{(1)}=0$. The QFI bound can therefore be written as

$$
F_{Q} \leq 4 T \min _{\left\{h_{00}^{(1)}, \mathbf{h}^{\left(\frac{1}{2}\right)}, \mathfrak{G}^{(0)}\right\}} \lambda, \quad \text { subject to } A \geq 0, \quad \beta^{(1)}=0 .
$$

The problem of determining the bounds is now fully specified as a semidefinite program using only the operators appearing in the master equation.

\section{APPENDIX B: TIGHTENING THE BOUND USING PHYSICAL CONSTRAINTS}

The bound in the form (11), which involves operator norms, may in some cases be tightened by taking into account additional physical constraints present in the problem considered.

\section{Superselection rules}

It is often the case in practice that the experimentalist does not have access to operations that create coherence between some sectors of the total Hilbert space. For example, there is no photonic experiment in which one is able to create, manipulate, or detect coherence between sectors of different total numbers of particles $n_{\mathrm{t}}=a_{1}^{\dagger} a_{1}+$ $a_{2}^{\dagger} a_{2}+\ldots$ in all modes (including local oscillators). This is a consequence of symmetries, like the time translation symmetry enforcing energy conservation in the example with particles. It is usually the case that the evolution, Eq. (1), does not create such coherences either, unless the evolution is "active" and describes the interaction of the system with a source. This can be formalized by introducing a map

$$
\mathcal{P}(\cdot)=\sum_{k} P_{k} \cdot P_{k}
$$

with $P_{k} P_{j}=\delta_{k, j} P_{k}$, which projects the state of the systems onto sectors that are eigenspaces of the conserved quantities labeled by $k$. Control operations and evolution are incoherent; they commute with map $\mathcal{P}$, implying, for instance, that $H$ is block diagonal, $\mathcal{P}(H)=H$.

Under such circumstances, QFI of a state $\rho$ might be overestimating the extractable classical Fisher information of the estimated parameter, as the information might be, for example, encoded in the coherence between different sectors. To get a tight expression, one then has to explicitly account for the conservation law by looking at QFI of the incoherent state $\mathcal{P}(\rho)$. A similar treatment can be done in our case. Since the projection map $\mathcal{P} \circ \mathcal{P}=\mathcal{P}$ commutes with both the controls and the evolution and has to be applied on the final state, we can explicitly account for the conservation law by considering the process $\tilde{\mathcal{E}}_{\omega}(t)$ where the free evolution induced by the master equation (1) is interjected with $\mathcal{P}$ after each infinitesimal time step $t$,

$\rho_{\omega}\left(t_{0}+t\right)=\tilde{\mathcal{E}}_{\omega}(t)\left(\rho\left(t_{0}\right)\right)=\mathcal{P} \circ \mathcal{E}_{\omega}(t) \circ \mathcal{P}\left(\rho\left(t_{0}\right)\right)$.

This composed channel $\tilde{\mathcal{E}}_{\omega}(d t)$ has a different set of Kraus operators:

$$
\begin{gathered}
K_{0 ; k}=P_{k}-P_{k}\left(\frac{1}{2} \mathbf{L}^{\dagger} \mathbf{L}+i H \omega\right) P_{k} t+O\left(t^{2}\right), \\
K_{j ; k \ell}=P_{k} L_{j} P_{\ell} \sqrt{t}+O\left(t^{3 / 2}\right) .
\end{gathered}
$$

But, as before, we can get the condition for the linearity of QFI as 


$$
\begin{aligned}
\beta^{(1)}= & H+\sum_{k} h_{00 ; k}^{(1)} P_{k}+\mathbf{h}_{k}^{\dagger\left(\frac{1}{2}\right)} P_{k} \mathbf{L} P_{k}+P_{k} \mathbf{L}^{\dagger} P_{k} \mathbf{h}_{k}^{\left(\frac{1}{2}\right)} \\
& +\sum_{k, \ell} P_{k} \mathbf{L}^{\dagger} \mathfrak{h}_{k \ell}^{(0)} P_{\ell} \mathbf{L} P_{k} .
\end{aligned}
$$

Note that because $H$ is block diagonal, we set all the entries of $h$ leading to terms of the form $P_{k} \cdot P_{\ell}$ with $(k \neq \ell)$ to zero. Hence, when the process is incoherent as described by the map $\mathcal{P}$, QFI scales linearly if each $H_{k}=P_{k} H P_{k}$ satisfies

$$
\begin{array}{r}
H_{k} \in \operatorname{span}\left\{P_{k},\left(P_{k} L_{j} P_{k}\right)^{H}, i\left(P_{k} L_{j} P_{k}\right)^{A H},\right. \\
\left.\left(P_{k} L_{j}^{\dagger} P_{\ell} L_{i} P_{k}\right)^{H},\left(P_{k} L_{j}^{\dagger} P_{\ell} L_{i} P_{k}\right)^{A H}\right\}
\end{array}
$$

for all $\ell, j$, and $i$.

Analogously, the minimization of the operator norm of $\alpha^{(1)}$ should be found for a block-diagonal operator:

$$
\begin{aligned}
\alpha^{(1)}= & \sum_{k \ell}\left(\mathbf{h}_{k}^{\left(\frac{1}{2}\right)} P_{k}+\mathfrak{h}_{k \ell}^{(0)} P_{\ell} \mathbf{L} P_{k}\right)^{\dagger} \\
& \times\left(\mathbf{h}_{k}^{\left(\frac{1}{2}\right)} P_{k}+\mathfrak{h}_{k \ell}^{(0)} P_{\ell} \mathbf{L} P_{k}\right) .
\end{aligned}
$$

\section{Restriction to a subspace}

The most obvious situation is when the system state is restricted to live in a particular subspace of the Hilbert space, and neither the Hamiltonian nor the noise operators $L_{i}$, nor the active control operations move the state out of this space. In this case, all operator norms appearing in Eq. (11), as well as conditions on vanishing operators $\beta$, should be understood as restricted to this subspace. For example, this is the case for atomic or optical systems with a fixed number of particles. Formally, let $P$ be the projection on the subspace. In all the formulas involving $\beta$ and $\alpha$ operators, we should simply replace all $H$ and $L_{i}$ by, respectively, $P H P$ and $P L_{i} P$. This situation can be viewed as a special case of the superselection rule situation when we leave only a projection on a single subspace.

\section{Fixing the number of particles in the protocol involving losses}

Let us consider the situation where we start with a state with a fixed number of particles which experiences losses as in the case of the models discussed in Sec. V. If we start with an $N$-atom state, then clearly, because of losses, we end up with a mixture of states with different atom numbers. Recall, however, that we work in the most general adaptive metrological scenario. We therefore assume that, at each adaptive step, we feed the system back with the lost atoms and carry on the evolution with an $\mathrm{N}$-atom state. In practice, this would amount to performing a nondemolition measurement of the number of remaining atoms; then, since every state of $n<N$ atoms can be isomorphically transcribed to the state of $N$ atoms, we do not lose any parameter information that was potentially present in the state as a result of earlier dynamics. With this in mind, and recalling that adaptive steps in the most general strategy can be chosen to be infinitesimally small, we can effectively think of this situation as an evolution with a fixed number of particles.

\section{Taking into account the state-time dependence}

Let us consider the situation where we have some additional knowledge of the form of the state of the system as it evolves in time under our protocol. Let us denote the general error-correction-assisted evolution by a completely positive trace preserving map $\mathcal{C}_{t}^{\omega}$. Let the initial state of the system plus ancilla be $\varrho_{0}=\left|\psi_{0}\right\rangle\left\langle\psi_{0}\right|$; then, the state at time $T$ is given by

$$
\varrho_{T}^{\omega}=\mathcal{C}_{T}^{\omega}\left(\varrho_{0}\right)=\sum_{\mathbf{i}} K_{\mathbf{i}}\left|\psi_{0}\right\rangle\left\langle\psi_{0}\right| K_{\mathbf{i}}^{\dagger},
$$

with some Kraus operators $K_{\mathbf{i}}$. We assume here that the error-correction protocol does not depend on time, but this is not crucial for the results that follow. QFI of any such channel satisfies

$$
F_{Q} \leq 4 \min _{\tilde{\boldsymbol{K}}} \operatorname{Tr} \sum_{\mathbf{i}} \dot{\tilde{K}}_{\mathbf{i}}\left|\psi_{0}\right\rangle\left\langle\psi_{0}\right| \dot{\tilde{K}}_{\mathbf{i}}^{\dagger}
$$

with the minimization over all Kraus representations of the channel $\tilde{\mathbf{K}} \simeq \mathbf{K}$. As we argued before, the channel can be decomposed into a product of infinitesimal channels $\mathcal{C}_{T}^{\omega}=\bigcirc_{n=1}^{m=T / d t} \mathcal{C}_{d t}^{\omega}$ so that a Kraus operator of the global overall process $K_{\mathbf{i}}=\prod_{n=1}^{m} K_{i_{n}}$. Thus, the derivative $\dot{K}^{T / d t}$ gives rise to a sum of $m$ terms, with each term having a derivative on an individual $\dot{K}_{i_{n}}$. This observation, together with the trace-preserving property of $\mathcal{C}_{t}^{\omega}$, allows us to rewrite the rhs of Eq. (B9) as

$$
\begin{aligned}
& 4 \int d t^{\prime} \operatorname{Tr} \sum_{i_{t^{\prime}}} \dot{\tilde{K}}_{i_{t^{\prime}}} \mathcal{E}_{\omega, t^{\prime}}\left(\varrho_{0}\right) \dot{\tilde{K}}_{i_{t^{\prime}}}^{\dagger} \\
& \quad+4 \int_{t^{\prime}>t^{\prime \prime}} d t^{\prime} d t^{\prime \prime} \operatorname{Tr} \sum_{i_{t^{\prime}}} \dot{\tilde{K}}_{i_{t^{\prime}}} \mathcal{C}_{t^{\prime}-t^{\prime \prime}}^{\omega}\left(\sum_{i_{t^{\prime \prime}}} \tilde{K}_{i_{t^{\prime \prime}}} \mathcal{C}_{t^{\prime \prime}}^{\omega}\left(\varrho_{0}\right) \dot{\tilde{K}}_{i_{t^{\prime \prime}}}\right) \tilde{K}_{i_{t^{\prime}}}^{\dagger} \\
& \quad+\text { H.c. }
\end{aligned}
$$

Choosing the local Kraus representation $\mathbf{K}$ such that the corresponding $\beta^{(1)}$ is set to zero at the order $d t$ (if this is possible) sets the last two terms to zero and gives the bound

$$
F_{Q} \leq 4 \int_{0}^{T} d t \operatorname{Tr}\left(\sum_{i_{t}} \dot{\tilde{K}}_{i_{t}}^{\dagger} \dot{\tilde{K}}_{i_{t}}\right) \mathcal{C}_{t}^{\omega}\left(\varrho_{0}\right)
$$

The terms in the parentheses are equal to $\alpha^{(1)}$, so we just obtained a state-dependent upper bound on QFI, 


$$
F_{Q} \leq 4 \int_{0}^{T} d t \min _{\tilde{\mathbf{K}} \text { s.t. } \beta^{(1)}=0} \operatorname{Tr} \alpha^{(1)}(t) \rho_{t}
$$

Note that this is in accordance with the general (stateindependent) bound, as for any state $\rho$, it is true that $\operatorname{Tr} \alpha^{(1)} \rho \leq\left\|\alpha^{(1)}\right\|$. Moreover, if one knows some properties of the time evolution of the state $\rho_{t}$ under the errorcorrection-assisted protocol, the bound in Eq. (B12) can be much tighter than the generic one $\left\|\alpha^{(1)}\right\| T$.

To give a specific example of the utility of such a bound, consider atomic interferometry in the presence of singleparticle losses as discussed in Sec. V. While deriving the bound, we assumed that lost atoms can be replaced with new ones. However, one might consider a more realistic situation where this is not the case and the lost atoms are not being replaced. For simplicity, let us assume that losses are state independent, $\gamma_{1}=\gamma_{2}=\gamma$. This means that, starting with a state of $N$ atoms, after time $t$, we will have an average number of atoms equal to $N e^{-\gamma t}$. Applying the time-dependent bound, and using Eq. (37) (which is also valid for incoherent mixtures of states with different numbers of atoms, where the mean number is $N$ ), we can therefore write

$$
F_{Q} \leq \int_{0}^{t} d t^{\prime} \frac{N e^{-\gamma t}}{\gamma}=\frac{N}{\gamma^{2}}\left(1-e^{-\gamma T}\right),
$$

which is, in general, tighter than $F_{Q} \leq N T / \gamma$.

\section{APPENDIX C: ERROR-CORRECTION SCHEME}

\section{Canonical form of noise operators}

If any of the noise operators $L_{j}$ are not traceless, they can be decomposed into $L_{j}=\lambda \rrbracket+\bar{L}_{j}$, with $\operatorname{Tr} 1 \bar{L}_{j}=0$. Under this substitution, the Lindblad operator remains the same (now denoted by $\bar{L}_{j}$ ), but an additional Hamiltonian term $-\mathrm{i}\left[\frac{\mathrm{i}}{2}\left(\lambda^{*} \bar{L}_{j}-\lambda \bar{L}_{j}^{\dagger}\right), \rho\right]$ appears in the master equation, which can be compensated by control operations. In addition, for any set of noise operators $\left\{L_{j}\right\}$, it is always possible to find an equivalent (i.e., giving rise to the same dynamics) set $\left\{\bar{L}_{j}\right\}$ of operators that are orthogonal [43] under the Hilbert-Schmidt product. Hence, one can always put the noise operators in the form $\operatorname{Tr} L_{j}=0$ and $\operatorname{Tr} L_{k}^{\dagger} L_{j}=0$ for all $j$ and $k$.

\section{Derivation of error-correction conditions (25)-(27)}

A general map $C$, which maps the system from the big Hilbert space $\mathcal{H}$ back onto a qubit subspace $\mathcal{H}_{Q}$, can be written as

$$
C(\cdot)=\sum_{\ell} R_{\ell} \cdot R_{\ell}^{\dagger}
$$

with $\quad R_{\ell}=\mu_{\ell}|\phi\rangle\left\langle\Phi_{\ell}\left|+\lambda_{\ell}\right| \xi\right\rangle\left\langle\Xi_{\ell}\right| \quad$ satisfying $\quad \mathbb{1}_{\mathcal{H}}=$ $\sum_{\ell} R_{\ell}^{\dagger} R_{\ell}=\sum_{\ell}\left(\left|\mu_{\ell}\right|^{2}\left|\Phi_{\ell}\right\rangle\left\langle\left.\Phi_{\ell}|+| \lambda_{\ell}\right|^{2} \mid \Xi_{\ell}\right\rangle\left\langle\Xi_{\ell}\right|\right)$. For our purpose, it is natural to consider the case where the two subspaces $\mathcal{H}_{\Phi}=\operatorname{span}\left\{\left|\Phi_{\ell}\right\rangle\right\}$ and $\mathcal{H}_{\Xi}=\operatorname{span}\left\{\left|\Xi_{\ell}\right\rangle\right\}$ are orthogonal, and the operator $\Pi_{\Xi}=\sum_{\ell}\left|\lambda_{\ell}\right|^{2}\left|\Xi_{\ell}\right\rangle\left\langle\Xi_{\ell}\right|$ and $\Pi_{\Phi}=\mathbb{1}_{\mathcal{H}}-\Pi_{\Xi}$ are orthogonal projectors. The map is then fully specified by the projector $\Pi_{\Xi}$ and the operator $M=\sum_{\ell} \mu_{\ell} \lambda_{\ell}^{*}\left|\Xi_{\ell}\right\rangle\left\langle\Phi_{\ell}\right|$. Given that $\operatorname{dim}\left(\mathcal{H}_{\Xi}\right) \leq \operatorname{dim}\left(\mathcal{H}_{\Phi}\right)$, it is possible to find a Kraus representation of the map $C$ for which the vectors $\lambda_{\ell}^{*}\left|\Xi_{\ell}\right\rangle$ are orthonormal; hence, we assume this result in the following [the same holds for $\mu_{\ell}^{*}\left|\Phi_{\ell}\right\rangle$ in the case $\left.\operatorname{dim}\left(\mathcal{H}_{\Xi}\right)>\operatorname{dim}\left(\mathcal{H}_{\Phi}\right)\right]$. Recall that we require the error-correction scheme to preserve the qubit subspace spanned by $|\phi\rangle$ and $|\xi\rangle$ under the action of the noise $\mathcal{L}$. For the map $C$, this implies

$$
\begin{gathered}
\operatorname{Tr} \Pi_{\Xi}(|\phi\rangle\langle\phi|+d t \mathcal{L}(|\phi\rangle\langle\phi|))=0, \\
\operatorname{Tr}_{\Xi}(|\xi\rangle\langle\xi|+d t \mathcal{L}(|\xi\rangle\langle\xi|))=1, \\
\operatorname{Tr}_{\Xi}(|\phi\rangle\langle\xi|+d t \mathcal{L}(|\phi\rangle\langle\xi|))=0, \\
\operatorname{Tr} M(|\phi\rangle\langle\phi|+d t \mathcal{L}(|\phi\rangle\langle\phi|))=0, \\
\operatorname{Tr} M(|\xi\rangle\langle\xi|+d t \mathcal{L}(|\xi\rangle\langle\xi|))=0, \\
\operatorname{Tr} M(|\phi\rangle\langle\xi|+d t \mathcal{L}(|\phi\rangle\langle\xi|))=1
\end{gathered}
$$

The first three equations imply that $|\phi\rangle \in \mathcal{H}_{\Phi}$ and $|\xi\rangle \in \mathcal{H}_{\Xi}$, as well as $L_{k}|\phi\rangle \in \mathcal{H}_{\Phi} \quad$ and $\quad L_{k}|\xi\rangle \in \mathcal{H}_{\Xi}$ for all $k$. Equations (C5) and (C6) impose, in addition, $\left\langle\phi\left|\mathbf{L}^{\dagger} \mathbf{L} M\right| \phi\right\rangle=\left\langle\xi\left|M \mathbf{L}^{\dagger} \mathbf{L}\right| \xi\right\rangle=0$. Finally, Eq. (C7) requires that $\operatorname{Tr} M|\phi\rangle\langle\xi|=1$, allowing us to write $M=|\xi\rangle\langle\phi|+M_{\perp}$, and

$\operatorname{Tr} M \sum_{k} L_{k}|\phi\rangle\langle\xi| L_{k}^{\dagger}-\frac{1}{2}\left(\left\langle\xi\left|\mathbf{L}^{\dagger} \mathbf{L}\right| \xi\right\rangle+\left\langle\phi\left|\mathbf{L}^{\dagger} \mathbf{L}\right| \phi\right\rangle\right)=0$.

This condition can be satisfied if and only if there is a unitary $U$ relating all the vectors $L_{k}|\phi\rangle$ to $L_{k}|\xi\rangle$. In this case, setting $M=U$ satisfies Eq. (C8). In turn, such a unitary exists if and only if one has

$$
\left\langle\phi\left|L_{k}^{\dagger} L_{j}\right| \phi\right\rangle=\left\langle\xi\left|L_{k}^{\dagger} L_{j}\right| \xi\right\rangle \quad \forall j, k ;
$$

i.e., the Gramm matrices for the two vector sets are the same.

In summary, an error-correction scheme that satisfies all of the requirements above exists if and only if one can find two states $|\phi\rangle$ and $|\xi\rangle$ such that

$$
\text { (a) }\langle\phi|H| \xi\rangle \neq 0
$$




$$
\begin{aligned}
& \text { (b) }\left\langle\phi\left|L_{k}^{\dagger} L_{j}\right| \xi\right\rangle=\left\langle\phi\left|L_{j}\right| \xi\right\rangle=0, \\
& \text { (c) }\left\langle\phi\left|L_{k}^{\dagger} L_{j}\right| \phi\right\rangle=\left\langle\xi\left|L_{k}^{\dagger} L_{j}\right| \xi\right\rangle,
\end{aligned}
$$

for all $k$ and $j$. Conditions (b) and (c) are properties of error correction, while (a) has to be satisfied in order to keep nontrivial unitary evolution in the qubit subspace $\mathcal{H}_{Q}$.

\section{APPENDIX D: DERIVATION OF THE FUNDAMENTAL PRECISION BOUND IN NONLINEAR METROLOGY WITH TWO-BODY LOSSES}

Starting from Eq. (40) and substituting $a_{1}^{\dagger 2} a_{1}^{2}$ with $n(n-1)$ and $a_{2}^{\dagger 2} a_{2}^{2}$ with $(N-n)(N-n-1)$, calculation of the operator norm amounts to maximization over $0 \leq n \leq N$. The bound (40) can therefore be rewritten as

$$
F_{Q} \leq \min _{\xi} \max _{n} \frac{T}{4}\left[(\xi-1)^{2} A+(\xi+1)^{2} B\right],
$$

where

$$
\begin{aligned}
& A=\frac{n(n-1)}{\gamma_{11}}+\frac{(N-n)(N-n-1)}{\gamma_{22}}, \\
& B=\frac{4(N-n) n}{\gamma_{12}} .
\end{aligned}
$$

Performing minimization over $\xi$ yields $\xi=(A-B) /$ $(A+B)$, and consequently,

$$
\begin{aligned}
F_{Q} \leq & \frac{N^{2} T}{\gamma_{12}} \max _{0 \leq x \leq 1}\left[\frac{1}{4(1-x) x}\right. \\
& \left.+\frac{1}{\frac{\gamma_{12}}{\gamma_{11}}\left(x-\frac{1}{N}\right) x++\frac{\gamma_{12}}{\gamma_{22}}(1-x)\left(1-x-\frac{1}{N}\right)}\right]^{-1},
\end{aligned}
$$

where we introduced $x=n / N, 0 \leq x \leq 1$. In the case $\gamma_{11}=\gamma_{22}$, this maximization can be performed analytically and results in

$$
F_{Q} \leq \frac{N^{2} T}{\gamma_{12}} \begin{cases}\frac{2(1-1 / N)}{(1+\sqrt{\lambda})^{2}} & \lambda \geq 1-\frac{2}{N} \\ \frac{1}{1+\lambda \frac{N}{N-2}} & \lambda<1-\frac{2}{N}\end{cases}
$$

where $\lambda=2 \gamma_{11} / \gamma_{12}$, and it yields Eq. (41) in the $N \gg 1$ limit. In the general case $\gamma_{11} \neq \gamma_{22}$, the maximization over $x$ can be easily performed numerically.

[1] V. Giovannetti, S. Lloyd, and L. Maccone, Advances in Quantum Metrology, Nat. Photonics 5, 222 (2011).

[2] G. Toth and I. Apellaniz, Quantum Metrology from a Quantum Information Science Perspective, J. Phys. A 47, 424006 (2014).
[3] R. Demkowicz-Dobrzanski, M. Jarzyna, and J. Kołodyński, Quantum Limits in Optical Interferometry, in Progress in Optics, edited by E. Wolf (Elsevier, New York, 2015), Vol. 60, pp. 345-435.

[4] L. Pezzè, A. Smerzi, M. K. Oberthaler, R. Schmied, and P. Treutlein, Non-Classical States of Atomic Ensembles: Fundamentals and Applications in Quantum Metrology, arXiv:1609.01609.

[5] R. Schnabel, Squeezed States of Light and Their Applications in Laser Interferometers, Phys. Rep. 684, 1 (2017).

[6] P. O. Schmidt, T. Rosenband, C. Langer, W. M. Itano, J. C. Bergquist, and D. J. Wineland, Spectroscopy Using Quantum Logic, Science 309, 749 (2005).

[7] LIGO Collaboration, A Gravitational Wave Observatory Operating Beyond the Quantum Shot-Noise Limit, Nat. Phys. 7, 962 (2011).

[8] B. Lücke, M. Scherer, J. Kruse, L. Pezzé, F. Deuretzbacher, P. Hyllus, O. Topic, J. Peise, W. Ertmer, J. Arlt, L. Santos, A. Smerzi, and C. Klempt, Twin Matter Waves for Interferometry Beyond the Classical Limit, Science 334, 773 (2011).

[9] M. A. Taylor, J. Janousek, V. Daria, J. Knittel, B. Hage, H.-A. Bachor, and W. P. Bowen, Biological Measurement Beyond the Quantum Limit, Nat. Photonics 7, 229 (2013).

[10] C. F. Ockeloen, R. Schmied, M. F. Riedel, and P. Treutlein, Quantum Metrology with a Scanning Probe Atom Interferometer, Phys. Rev. Lett. 111, 143001 (2013).

[11] T. Kovachy, P. Asenbaum, C. Overstreet, C. A. Donnelly, S. M. Dickerson, A. Sugarbaker, J. M. Hogan, and M. A. Kasevich, Quantum Superposition at the Half-Metre Scale, Nature (London) 528, 530 (2015).

[12] B. Barrett, L. Antoni-Micollier, L. Chichet, B. Battelier, T. Lévèque, A. Landragin, and P. Bouyer, Dual Matter-Wave Inertial Sensors in Weightlessness, Nat. Commun. 7, 13786 (2016).

[13] C. M. Caves, Quantum-Mechanical Noise in an Interferometer, Phys. Rev. D 23, 1693 (1981).

[14] S. L. Braunstein, Quantum Limits on Precision Measurements of Phase, Phys. Rev. Lett. 69, 3598 (1992).

[15] D. W. Berry and H. M. Wiseman, Optimal States and Almost Optimal Adaptive Measurements for Quantum Interferometry, Phys. Rev. Lett. 85, 5098 (2000).

[16] V. Giovannetti, S. Lloyd, and L. Maccone, Quantum Metrology, Phys. Rev. Lett. 96, 010401 (2006).

[17] S. F. Huelga, C. Macchiavello, T. Pellizzari, A. K. Ekert, M. B. Plenio, and J. I. Cirac, Improvement of Frequency Standards with Quantum Entanglement, Phys. Rev. Lett. 79, 3865 (1997).

[18] A. Fujiwara and H. Imai, A Fibre Bundle over Manifolds of Quantum Channels and Its Application to Quantum Statistics, J. Phys. A 41, 255304 (2008).

[19] M. G. Genoni, S. Olivares, and M. G. A. Paris, Optical Phase Estimation in the Presence of Phase Diffusion, Phys. Rev. Lett. 106, 153603 (2011).

[20] B. M. Escher, R. L. de Matos Filho, and L. Davidovich, General Framework for Estimating the Ultimate Precision Limit in Noisy Quantum-Enhanced Metrology, Nat. Phys. 7, 406 (2011). 
[21] S. I. Knysh, E. H. Chen, and G. A. Durkin, True Limits to Precision via Unique Quantum Probe, arXiv: 1402.0495.

[22] R. Demkowicz-Dobrzański, J. Kołodyński, and M. Guţă, The Elusive Heisenberg Limit in Quantum-Enhanced Metrology, Nat. Commun. 3, 1063 (2012).

[23] R. Demkowicz-Dobrzański, K. Banaszek, and R. Schnabel, Fundamental Quantum Interferometry Bound for the Squeezed-Light-Enhanced Gravitational Wave Detector GEO 600, Phys. Rev. A 88, 041802(R) (2013).

[24] M. Jarzyna and M. Zwierz, Parameter Estimation in the Presence of the Most General Gaussian Dissipative Reservoir, Phys. Rev. A 95, 012109 (2017).

[25] R. Demkowicz-Dobrzański and L. Maccone, Using Entanglement Against Noise in Quantum Metrology, Phys. Rev. Lett. 113, 250801 (2014).

[26] P. Sekatski, M. Skotiniotis, J. Kołodyński, and W. Dür, Quantum Metrology with Full and Fast Quantum Control, Quantum 1, 27 (2017).

[27] H.-P. Breuer and F. Petruccione, The Theory of Open Quantum Systems (Oxford University Press, New York, 2002).

[28] S. Boixo, S. T. Flammia, C. M. Caves, and J. M. Geremia, Generalized Limits for Single-Parameter Quantum Estimation, Phys. Rev. Lett. 98, 090401 (2007).

[29] A. Luis, Quantum Limits, Nonseparable Transformations, and Nonlinear Optics, Phys. Rev. A 76, 035801 (2007).

[30] S. Boixo, A. Datta, M. J. Davis, S. T. Flammia, A. Shaji, and C. M. Caves, Quantum Metrology: Dynamics Versus Entanglement, Phys. Rev. Lett. 101, 040403 (2008).

[31] S. Choi and B. Sundaram, Bose-Einstein Condensate as a Nonlinear Ramsey Interferometer Operating Beyond the Heisenberg Limit, Phys. Rev. A 77, 053613 (2008).

[32] M. Napolitano and M. W. Mitchell, Nonlinear Metrology with a Quantum Interface, New J. Phys. 12, 093016 (2010).

[33] C. Gross, T. Zibold, E. Nicklas, J. Esteve, and M. K. Oberthaler, Nonlinear Atom Interferometer Surpasses Classical Precision Limit, Nature (London) 464, 1165 (2010).

[34] M. J. W. Hall and H. M. Wiseman, Does Nonlinear Metrology Offer Improved Resolution? Answers from Quantum Information Theory, Phys. Rev. X 2, 041006 (2012).

[35] J. Joo, K. Park, H. Jeong, W. J. Munro, K. Nemoto, and T. P. Spiller, Quantum Metrology for Nonlinear Phase Shifts with Entangled Coherent States, Phys. Rev. A 86, 043828 (2012).

[36] R. J. Sewell, M. Napolitano, N. Behbood, G. Colangelo, F. M. Ciurana, and M. W. Mitchell, Ultrasensitive Atomic Spin Measurements with a Nonlinear Interferometer, Phys. Rev. X 4, 021045 (2014).

[37] M. Beau and A. del Campo, Nonlinear Quantum Metrology of Many-Body Open Systems, Phys. Rev. Lett. 119, 010403 (2017).

[38] M. Hotta, T. Karasawa, and M. Ozawa, Ancilla-Assisted Enhancement of Channel Estimation for Low-Noise Parameters, Phys. Rev. A 72, 052334 (2005).
[39] M. Hotta, T. Karasawa, and M. Ozawa, N-Body-Extended Channel Estimation for Low-Noise Parameters, J. Phys. A 39, 14465 (2006).

[40] J. Kołodyński and R. Demkowicz-Dobrzański, Efficient Tools for Quantum Metrology with Uncorrelated Noise, New J. Phys. 15, 073043 (2013).

[41] M. Takeoka and M. M. Wilde, Optimal Estimation and Discrimination of Excess Noise in Thermal and Amplifier Channels, arXiv:1611.09165.

[42] S. Pirandola and C. Lupo, Ultimate Precision of Adaptive Noise Estimation, Phys. Rev. Lett. 118, 100502 (2017).

[43] P. Pearle, Simple Derivation of the Lindblad Equation, Eur. J. Phys. 33, 805 (2012).

[44] M. J. W. Hall, J. D. Cresser, L. Li, and E. Andersson, Canonical Form of Master Equations and Characterization of Non-Markovianity, Phys. Rev. A 89, 042120 (2014).

[45] E. Knill and R. Laflamme, Theory of Quantum ErrorCorrecting Codes, Phys. Rev. A 55, 900 (1997).

[46] C. Bény, Perturbative Quantum Error Correction, Phys. Rev. Lett. 107, 080501 (2011).

[47] G. Arrad, Y. Vinkler, D. Aharonov, and A. Retzker, Increasing Sensing Resolution with Error Correction, Phys. Rev. Lett. 112, 150801 (2014).

[48] E. M. Kessler, I. Lovchinsky, A. O. Sushkov, and M. D. Lukin, Quantum Error Correction for Metrology, Phys. Rev. Lett. 112, 150802 (2014).

[49] W. Dür, M. Skotiniotis, F. Fröwis, and B. Kraus, Improved Quantum Metrology Using Quantum Error Correction, Phys. Rev. Lett. 112, 080801 (2014).

[50] S. Zhou, M. Zhang, J. Preskill, and L. Jiang, Achieving the Heisenberg Limit in Quantum Metrology Using Quantum Error Correction, arXiv:1706.02445.

[51] J. Kolodynski and R. Demkowicz-Dobrzanski, Phase Estimation without a priori Phase Knowledge in the Presence of Loss, Phys. Rev. A 82, 053804 (2010).

[52] S. Knysh, V. N. Smelyanskiy, and G. A. Durkin, Scaling Laws for Precision in Quantum Interferometry and the Bifurcation Landscape of the Optimal State, Phys. Rev. A 83, 021804 (2011).

[53] J. Czajkowski and R. Demkowicz-Dobrzanski (unpublished).

[54] P. M. Anisimov, G. M. Raterman, A. Chiruvelli, W. N. Plick, S. D. Huver, H. Lee, and J. P. Dowling, Quantum Metrology with Two-Mode Squeezed Vacuum: Parity Detection Beats the Heisenberg Limit, Phys. Rev. Lett. 104, 103602 (2010).

[55] M. Tsang, Ziv-Zakai Error Bounds for Quantum Parameter Estimation, Phys. Rev. Lett. 108, 230401 (2012).

[56] D. W. Berry, M. J. W. Hall, M. Zwierz, and H. M. Wiseman, Optimal Heisenberg-Style Bounds for the Average Performance of Arbitrary Phase Estimates, Phys. Rev. A 86, 053813 (2012).

[57] V. Giovannetti and L. Maccone, Sub-Heisenberg Estimation Strategies Are Ineffective, Phys. Rev. Lett. 108, 210404 (2012).

[58] M. Jarzyna and R. Demkowicz-Dobrzański, True Precision Limits in Quantum Metrology, New J. Phys. 17, 013010 (2015). 
[59] A. W. Chin, S. F. Huelga, and M. B. Plenio, Quantum Metrology in Non-Markovian Environments, Phys. Rev. Lett. 109, 233601 (2012).

[60] A. Smirne, J. Kołodyński, S. F. Huelga, and R. DemkowiczDobrzański, Ultimate Precision Limits for Noisy Frequency Estimation, Phys. Rev. Lett. 116, 120801 (2016).
[61] P. Sekatski, M. Skotiniotis, and W. Dür, Dynamical Decoupling Leads to Improved Scaling in Noisy Quantum Metrology, New J. Phys. 18, 073034 (2016).

[62] H. Yuan, Ultimate Precision for Simultaneously Estimating Multiple Components of a Magnetic Field, arXiv:1601 .04466 [Phys. Rev. Lett. (to be published)]. 